Document downloaded from:

http://hdl.handle.net/10251/51310

This paper must be cited as:

Fernández Fernández, I.; Gozálvez Zafrilla, JM.; Santafé Moros, MA. (2014). Efficient fitting of nanofiltration model parameters for a specified groundwater type by selecting suitable characterization data-sets. Desalination and Water Treatment. 1-10. doi:10.1080/19443994.2014.946714.

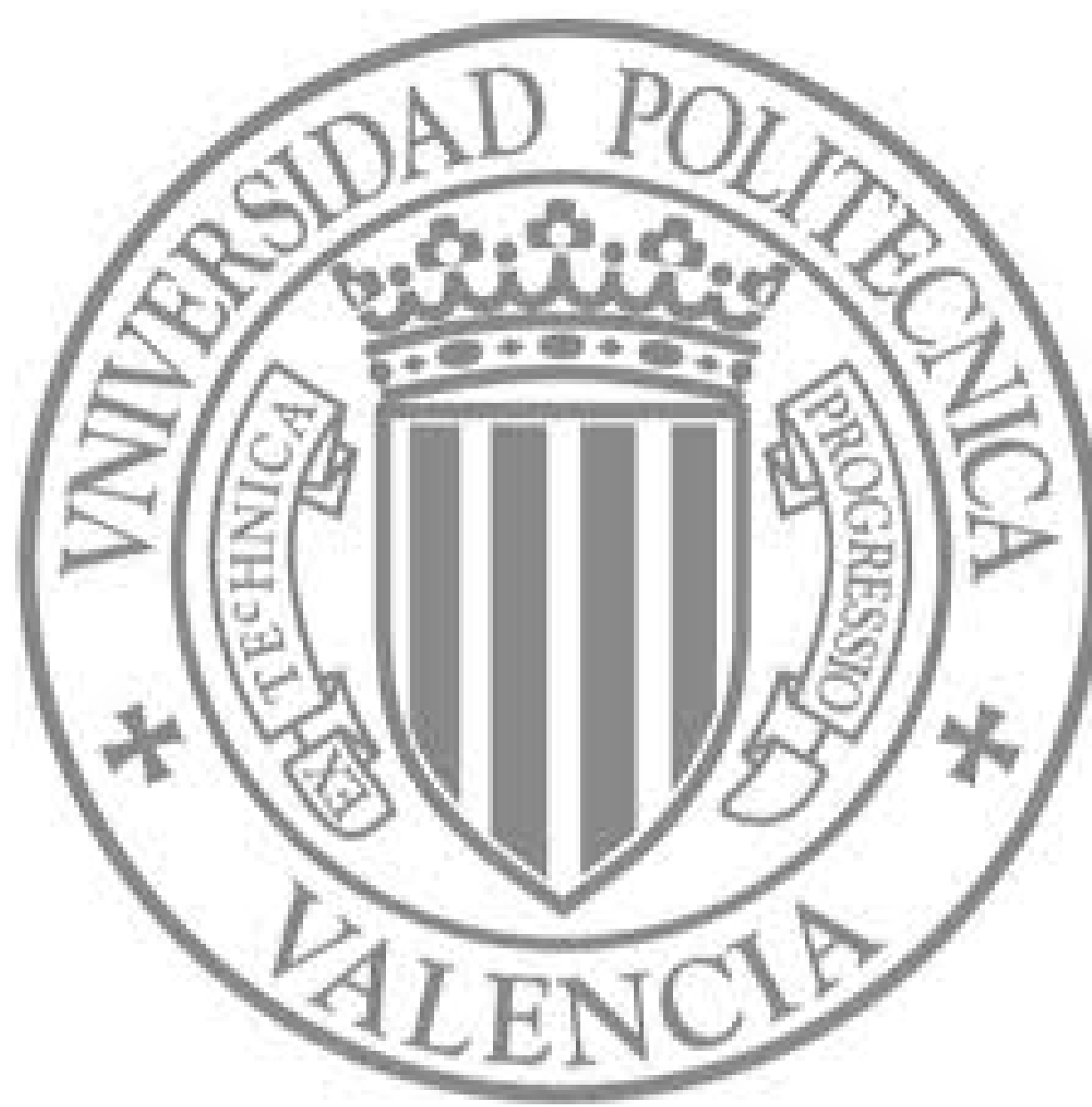

The final publication is available at

http://dx.doi.org/10.1080/19443994.2014.946714

Copyright Taylor \&amp;amp; Francis: STM, Behavioural Science and Public Health Titles 


\title{
Efficient fitting of nanofiltration model parameters for a specified groundwater type by selecting suitable characterization data sets
}

\author{
Irene Fernández-Fernández, José M. Gozálvez-Zafrilla, Asunción Santafé-Moros \\ Institute for Industrial, Radiophysical and Environmental Safety (ISIRYM), \\ Universitat Politècnica de Valencia, C/ Camino de Vera s/n, 46022 Valencia (Spain)
}

Keywords: nanofiltration, DSPM-DE model, model fitting

\begin{abstract}
Nanofiltration models can be useful to perform optimal designs of membrane systems and to estimate membrane performance for waters. There is special interest in obtaining nanofiltration models with parameters based on measurable properties of the membrane and independent from feed and operating conditions. However, many times, from a practical point of view, nanofiltration parameters can be directly fitted from experiments performed with salts in a range of compositions.

The aim of this study is to select the better combination of experiments yielding to a suitable fitting for the nanofiltration model DSPM-DE. In our case, the best fitting for a specific group of waters is searched (groundwater belonging to a Mediterranean region with moderate salinity).

The first part of the work is devoted to study which combinations of salts and concentrations lead to higher information. Using known values of nanofiltration parameters, permselective results were computationally generated using the nanofiltration model for a huge number of different combinations of compositions and random parameters sets. Performance factors for permeate flux and rejection based on the comparison between the characterization groups and a control group were defined. The second part of the work focused on the experimental validation of the selection procedure.

The results showed that there are characterization sets (composition and operating conditions) that yield higher fitting performance. These combinations of experiments should be the preferred ones to be performed when direct fitting from experiments is going to be performed.
\end{abstract}

\section{Introduction}

Nanofiltration (NF) has shown its effectiveness on the ion removal from water, being an effective technique for the treatment of brackish waters to obtain drinking-water or reuse industrial wastewater. To develop nanofiltration processes, the knowledge of factors affecting separation is essential but obtaining precise and reliable experimental data is a long and resource intensive process. The development of NF models is fundamental to facilitate this knowledge. Besides, the developed NF models can be a useful tool for design and operation of NF systems.

Several attempts have been performed for modelling NF separation processes during the last decades. The most successful models are those derived from the Donnan steric-partitioning pore model (DSPM) developed by Bowen and Mukhtar [1]. These models use the extended Nernst-Planck equation to describe ionic transport across the membrane. Afterwards, the model was updated by Bowen and Welfoot [2] including dielectric exclusion, the dependence of chemical potential on pressure on solute transport and an increased solvent viscosity within the pores (DSPM-DE).

This model considers four characteristic parameters of the membrane: membrane pore size $\left(r_{p}\right)$, the ratio of membrane thickness to effective porosity $\left(\Delta x / A_{k}\right)$, the membrane charge density $(\chi d)$ and the dielectric constant of the membrane $(\varepsilon)$. The pore size of the membrane can be determined directly by liquid-liquid porosimetry or atomic force microscopy [3], or indirectly by nanofiltration of uncharged solutes [4]. Then, the $\Delta x / A_{k}$ parameter can be calculated with Eq. (1) with the value of permeability to pure water $\left(L_{p, w}\right)$ obtained with experiments carried out at various transmembrane pressures. The membrane charge density is very influenced by feed composition $\left(C_{T}\right)$ so it is usually correlated using a potential function (2). Also, these parameters could be obtained directly using a fitting procedure of the DSPM-DE model to experimental data.

$L_{p, w}=\frac{r_{p}^{2}}{8 \cdot \mu \cdot \Delta x / A_{k}}$

$\chi_{d}=\chi_{d q} \cdot C_{T}^{\chi_{d s}}$

However, nanofiltration parameters are not completely independent from composition [5]. This implies the convenience of using, in the fitting procedure, model solutions with composition as similar as possible to the waters that will be treated by the membrane. Another issue that must be taken into account 
is the determination of the right experiments to be used in the fitting procedure of the parameters. The determination of the best combinations of experiments for a specific type of waters would constitute a significant improvement of the quality of the further model predictions.

The aim of this work is to find an optimal set of experiments to fit the model in order to obtain the best predictions in a given a range of water compositions. By an optimal set of experiments is meant a subset with a number of experiments not too high to be time consuming and with not too complex feed compositions. Additionally, the model parameters obtained from the optimal subset must be representative of the water type on which the model will be applied.

To illustrate the procedure, a specific type of natural groundwater present in a Mediterranean region of Spain (Valencian Community) was selected as universe of study. According to the information collected in [6], these waters are characterized by low salinity levels, between 500 and $1500 \mathrm{mg} / \mathrm{L}$. Major ions are calcium, sulfate, chloride and bicarbonate. These waters have nitrate levels that slightly exceed the legal limit $(50 \mathrm{mg} / \mathrm{L})$, being nanofiltration a potential technique to treat them to obtain drinking-water. Moreover, coming from diverse rocks, the concentrations of some ions are slightly correlated. A classic experimental design does not take into account this correlation between ions; therefore, a better approach can be to perform random experiments in the composition range of interest.

The most direct approach to perform the selection of the best combinations of experiments intended for fitting would be to compare the real performance of the membrane for the brackish waters group with the model predictions corresponding to parameters fitted from different groups of characterization experiments. So, the nanofiltration of a representative set of the universe of natural brackish waters studied would be performed experimentally, obtaining total rejection and permeate flux data. Afterwards, a large number of experimental sets with different feed compositions would be chosen to be tested under the same operating conditions in order to obtain different parameter sets. Finally, each set of parameters would be used to simulate the nanofiltration of natural waters and the obtained results would be compared with the experimental ones to evaluate the corresponding error.

However, the latter procedure implies a large number of experiments, so it was decided to carry out a preliminary study using simulation and, then, check the obtained results in a posterior experimental phase.

Besides, as the fitting process takes much more time than the calculation procedure, a method using direct evaluation is proposed. The method is based on the use of two different kinds of group representative of the universe of waters to be studied:

- The control group formed by randomly sampled waters from the universe of study.

- The characterization group, any of the combinations of fitting experiments to be assessed.

It is assumed that a good group of fitting experiments will properly predict the control group if the variations of the model parameters equally affect both groups. Therefore, the model parameters will be varied in a range and the sensitivity to change of both groups will be evaluated.

For the conclusions drawn from the comparison to have validity, it is essential that the items assigned to both groups be representative of the universe of study.

This paper is structured as follows: first, the calculation tool used to fit the DSPM-DE model and to simulate the nanofiltration performance is described. Then, the correlation between the control group and different characterization groups is explained and the characterization group that better predicts the total rejection of the control group is detailed. Finally, the results of the experimental testing of this characterization group with a validation case are exposed.

\section{Methodology}

\subsection{Calculation tool}

Calculations with the DSPM-DE model were performed using a code implemented in MATLAB. The mentioned program has two different operation modes: calculation and fitting.

The calculation mode is used to simulate the nanofiltration performance for given values of $r_{p}, \Delta x / A_{k}$, $\chi d q, \chi d s$ and $\varepsilon$. The permeability to pure water and the thickness of the boundary layer can be included as additional parameters. In calculation mode, feed molar composition and operating conditions (pressure, temperature, cross-flow velocity and feed $\mathrm{pH}$ ) must be specified. The program returns the composition and volumetric flux of the permeate stream. It is also possible to estimate the pressure required to obtain a specified permeate flux.

The fitting mode is used to obtain the model parameters from a set of experimental nanofiltration data. In this mode, besides feed molar composition and operating conditions, the volumetric flux and ion concentration of the permeate stream must be introduced. With an initial value introduced for each parameter, a genetic algorithm is used to perform a global optimization of the parameters followed by a 
local optimization using a gradient-based method. Required computational parameters of the algorithm are the number of generations, population size, mutation rate and crossover.

\subsection{Selection of the optimal group of experiments for fitting}

In order to select the best set of cases which leads to an optimal prediction of the filtration behavior of a membrane, the simulation results of the model for two types of groups were compared:

- The control group was formed by randomly sampling 58 waters from the universe of study.

- The different characterization groups were selected combinations of salt solutions of variable complexity, different concentrations and experimental conditions, also meeting these requirements: i) the composition of experiments performed must have limited complexity, ii) the range of composition of the solutions used must fall in the composition range of the universe of waters of study.

According to Wilks formula [7], the number of Monte Carlo runs to perform an uncertainty and sensitivity study in order to get a probability content of 0.95 at a confidence level of 0.95 is 93 . Therefore 93 random combinations of the model parameters were performed. To do that values of the parameters were independently generated using uniform probability distributions functions around a reference value. According to precedent data for commercial nanofiltration membranes in Table 1, the selected range for each of the 93 sets of model parameters is shown in Table 2. The $\Delta x / A_{k}$ values were calculated with Eq. (1), based on those of the pore radius and water permeability. The values of the coefficients of Eq. (2) for the charge density were taken to obtain typical charge values according to the total concentrations used in the simulation (between 13 and $42 \mathrm{mN}$ ).

The complete procedure is schematized in Fig. 1.

Table 1. Characteristic parameters of different commercial membranes in literature.

\begin{tabular}{|c|c|c|c|c|}
\hline Membrane & $\mathbf{r}_{\mathbf{p}}(\mathbf{n m})$ & $\Delta \mathbf{x} / \mathbf{A}_{\mathbf{x}}(\boldsymbol{\mu m})$ & $\varepsilon$ & Reference \\
\hline \multirow{2}{*}{ Desal-5 DL (GE-Osmonics) } & 0.5 & 0.37 & & {$[8]$} \\
\hline & 0.45 & 2.54 & & [9] \\
\hline Desal-5 DK(GE-Osmonics) & $0.42-0.44$ & $2.59-2.74$ & & [9] \\
\hline \multirow{2}{*}{ Desal DK (GE-Osmonics) } & & $1.53-2.99$ & & {$[2]$} \\
\hline & & $2.76-3.89$ & & [10] \\
\hline Desal G-5 (GE-Osmonics) & 0.84 & 40.54 & & [9] \\
\hline Desal G-10 (GE-Osmonics) & 1.3 & & & [11] \\
\hline Desal G-20 (GE-Osmonics) & 1.2 & & & [11] \\
\hline Desal HL (GE-Osmonics) & & & $33-34-35$ & [12] \\
\hline ESNA (Hydranautics) & $0.39-0.41-0.43$ & & & [13] \\
\hline ESNA1 (Hydranautics) & 0.3 & & & [14] \\
\hline ESNA1-K1 (Hydranautics) & 0.47 & & & [15] \\
\hline ESNA1-LF (Hydranautics) & 0.3 & & & [14] \\
\hline \multirow{2}{*}{ NF (DOW-Filmtec) } & 0.43 & 0.26 & & {$[8]$} \\
\hline & $0.43-0.44$ & $2.47-2.62$ & & [9] \\
\hline NF 70 (DOW-Filmtec) & $0.41-0.44-0.6-0.48$ & & & [16] \\
\hline NF 250 (DOW-Filmtec) & & & $41-41.5-42.4$ & [4] \\
\hline \multirow{2}{*}{ NF270 (DOW-Filmtec) } & 0.43 & 0.45 & & {$[8]$} \\
\hline & $0.43-0.54-0.65-0.54$ & & & [16] \\
\hline
\end{tabular}


Table 2. Variation range of the model parameters: pore size $\left(r_{p}\right)$, effective porosity $\left(\Delta x / A_{k}\right)$, permeability to pure water $\left(L_{p, w}\right)$, the terms of the charge equation $\left(\chi_{d q}\right.$ and $\left.\chi_{d s}\right)$ and the dielectric constant $(\varepsilon)$.

\begin{tabular}{lccc}
\hline & Minimum & Maximum & Reference \\
\hline $\mathbf{r}_{\mathbf{p}}(\mathbf{n m})$ & 0.39 & 0.60 & 0.49 \\
$\boldsymbol{\Delta} \mathbf{x} / \mathbf{A}_{\mathbf{k}}(\boldsymbol{\mu m})$ & 0.33 & 1.90 & 1.11 \\
$\mathbf{L}_{\mathbf{p}, \mathbf{w}}\left(\mathbf{L} \cdot \mathbf{m}^{\mathbf{2}} \cdot \mathbf{s}^{-\mathbf{1}} \cdot \mathbf{b a r}^{-\mathbf{1}}\right)$ & 8 & 24 & 16 \\
$\boldsymbol{\chi}_{\mathbf{d q}}$ & -0.5 & -0.1 & -0.3 \\
$\boldsymbol{\chi}_{\mathbf{d s}}$ & 0.9 & 1.5 & 1.2 \\
$\boldsymbol{\varepsilon}$ & 33 & 43 & 38 \\
\hline
\end{tabular}

\section{PERTURBATION}

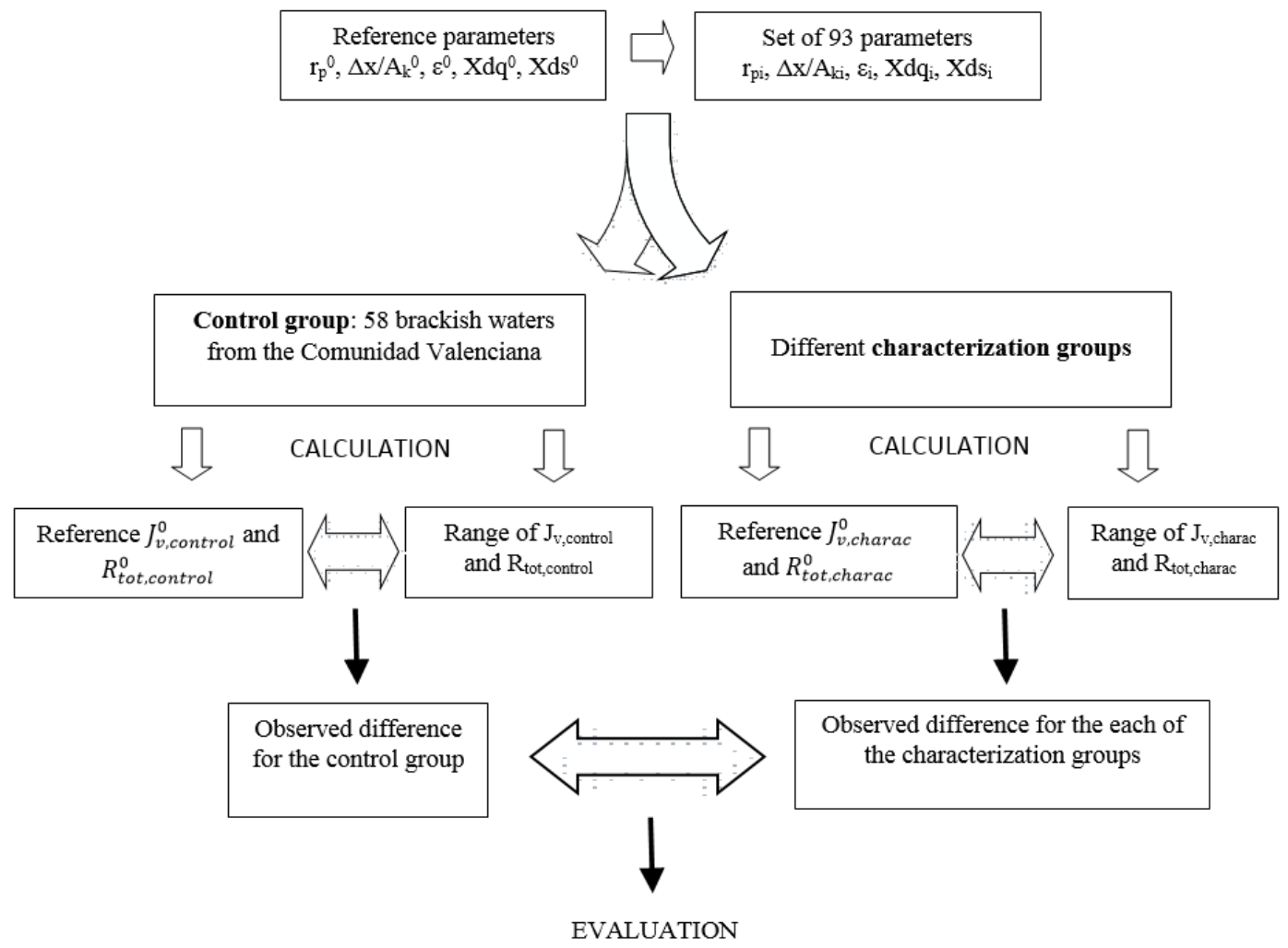

Fig. 1. Schema of the working process to study the correlation within the control group and the characterization groups.

In the definition of the control group compositions only major ions were taken into account, that is, $\mathrm{Ca}^{2+}, \mathrm{Mg}^{2+}, \mathrm{Na}^{+}$and $\mathrm{K}^{+}$and $\mathrm{Cl}^{-}, \mathrm{NO}_{3}{ }^{-}, \mathrm{HCO}_{3}{ }^{-}, \mathrm{SO}_{4}{ }^{2-}$ and $\mathrm{CO}_{3}{ }^{2-}$. The concentration ranges for each type of ion are shown in Table 3. Three operating pressures of 5, 10, and 15 bar were considered as representative of the typical pressure range for the nanofiltration of the waters. Temperature, cross flow velocity and $\mathrm{pH}$ were fixed at $25^{\circ} \mathrm{C}, 2 \mathrm{~m} / \mathrm{s}$ and 6 respectively.

For the sake of simplicity, the characterization groups were formed considering solutions constituted

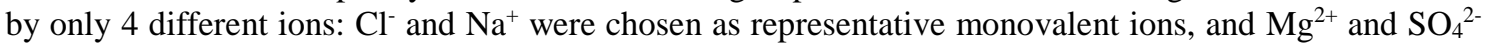
as representative of the divalent ones because of their important presence in the natural brackish waters studied. The $\mathrm{Ca}^{2+}$ cation was discarded to avoid potential scaling problems in the experimental tests. Two levels of total feed concentration were fixed: 13 and $42 \mathrm{mN}$, corresponding to the maximum and minimum values in the control group. The operating pressure was established at two levels (5 and 15 bar). The other operating variables were fixed at the same values used in the control group $\left(T=25^{\circ} \mathrm{C}, p H=6\right.$, $v=2 \mathrm{~m} / \mathrm{s}$ ). Combining the different levels of total concentration, ion composition and operating pressure, a total of 36 different cases was obtained (Table 4). 
Table 3. Concentration range for the selected ions on the control group.

\begin{tabular}{lcc|ccc}
\hline & \multicolumn{5}{c}{ Concentration $(\mathbf{m M})$} \\
Minimum & Maximum & \multicolumn{1}{c}{ Minimum } & Maximum \\
\hline $\mathbf{C a}^{2+}$ & 1.90 & 5.01 & $\mathbf{C l}^{-}$ & 0.44 & 6.62 \\
$\mathbf{M g}^{2+}$ & 0.13 & 3.74 & $\mathbf{N O}_{3}{ }^{-}$ & 0.83 & 1.60 \\
$\mathbf{N a}^{+}$ & 0.44 & 6.43 & $\mathbf{H C O}_{3}{ }^{-}$ & 1.83 & 6.83 \\
$\mathbf{K}^{+}$ & 0.02 & 0.13 & $\mathbf{S O}_{4}{ }^{2-}$ & 0.54 & 4.76 \\
& & $\mathbf{C O}_{3}{ }^{2-}$ & 0.02 & 0.11 \\
\hline
\end{tabular}

Table 4. Cases defined to build the characterization groups.

\begin{tabular}{ccccccc|ccccccc}
\hline Case & $\begin{array}{c}\mathbf{C}_{\mathbf{T}} \\
(\mathbf{m N})\end{array}$ & $\begin{array}{c}\mathbf{P} \\
(\mathbf{b a r})\end{array}$ & $\mathbf{X}_{\mathbf{M g} 2+}$ & $\mathbf{X}_{\mathbf{N a}+}$ & $\mathbf{X}_{\mathbf{S O 4 2 -}-}$ & $\mathbf{X}_{\mathbf{C l}-}$ & $\mathbf{C a s e}$ & $\begin{array}{c}\mathbf{C}_{\mathbf{T}} \\
(\mathbf{m N})\end{array}$ & $\begin{array}{c}\mathbf{P} \\
(\mathbf{b a r})\end{array}$ & $\mathbf{X}_{\mathbf{M g 2}+}$ & $\mathbf{X}_{\mathbf{N a}+}$ & $\mathbf{X}_{\mathbf{S O 4 2 -}}$ & $\mathbf{X}_{\mathbf{C l}}$ \\
\hline $\mathbf{1}$ & 13 & 5 & 0 & 1 & 1 & 0 & $\mathbf{1 9}$ & 42 & 5 & 0 & 1 & 1 & 0 \\
$\mathbf{2}$ & 13 & 5 & 0 & 1 & 0.5 & 0.5 & $\mathbf{2 0}$ & 42 & 5 & 0 & 1 & 0.5 & 0.5 \\
$\mathbf{3}$ & 13 & 5 & 0 & 1 & 0 & 1 & $\mathbf{2 1}$ & 42 & 5 & 0 & 1 & 0 & 1 \\
$\mathbf{4}$ & 13 & 5 & 0.5 & 0.5 & 1 & 0 & $\mathbf{2 2}$ & 42 & 5 & 0.5 & 0.5 & 1 & 0 \\
$\mathbf{5}$ & 13 & 5 & 0.5 & 0.5 & 0.5 & 0.5 & $\mathbf{2 3}$ & 42 & 5 & 0.5 & 0.5 & 0.5 & 0.5 \\
$\mathbf{6}$ & 13 & 5 & 0.5 & 0.5 & 0 & 1 & $\mathbf{2 4}$ & 42 & 5 & 0.5 & 0.5 & 0 & 1 \\
$\mathbf{7}$ & 13 & 5 & 1 & 0 & 1 & 0 & $\mathbf{2 5}$ & 42 & 5 & 1 & 0 & 1 & 0 \\
$\mathbf{8}$ & 13 & 5 & 1 & 0 & 0.5 & 0.5 & $\mathbf{2 6}$ & 42 & 5 & 1 & 0 & 0.5 & 0.5 \\
$\mathbf{9}$ & 13 & 5 & 1 & 0 & 0 & 1 & $\mathbf{2 7}$ & 42 & 5 & 1 & 0 & 0 & 1 \\
$\mathbf{1 0}$ & 13 & 15 & 0 & 1 & 1 & 0 & $\mathbf{2 8}$ & 42 & 15 & 0 & 1 & 1 & 0 \\
$\mathbf{1 1}$ & 13 & 15 & 0 & 1 & 0.5 & 0.5 & $\mathbf{2 9}$ & 42 & 15 & 0 & 1 & 0.5 & 0.5 \\
$\mathbf{1 2}$ & 13 & 15 & 0 & 1 & 0 & 1 & $\mathbf{3 0}$ & 42 & 15 & 0 & 1 & 0 & 1 \\
$\mathbf{1 3}$ & 13 & 15 & 0.5 & 0.5 & 1 & 0 & $\mathbf{3 1}$ & 42 & 15 & 0.5 & 0.5 & 1 & 0 \\
$\mathbf{1 4}$ & 13 & 15 & 0.5 & 0.5 & 0.5 & 0.5 & $\mathbf{3 2}$ & 42 & 15 & 0.5 & 0.5 & 0.5 & 0.5 \\
$\mathbf{1 5}$ & 13 & 15 & 0.5 & 0.5 & 0 & 1 & $\mathbf{3 3}$ & 42 & 15 & 0.5 & 0.5 & 0 & 1 \\
$\mathbf{1 6}$ & 13 & 15 & 1 & 0 & 1 & 0 & $\mathbf{3 4}$ & 42 & 15 & 1 & 0 & 1 & 0 \\
$\mathbf{1 7}$ & 13 & 15 & 1 & 0 & 0.5 & 0.5 & $\mathbf{3 5}$ & 42 & 15 & 1 & 0 & 0.5 & 0.5 \\
$\mathbf{1 8}$ & 13 & 15 & 1 & 0 & 0 & 1 & $\mathbf{3 6}$ & 42 & 15 & 1 & 0 & 0 & 1 \\
\hline
\end{tabular}

For the control and each one of the characterization groups, once established the feed composition and the operating conditions, permeate fluxes and ion concentrations were calculated using the MATLAB code for all the parameter sets.

Two, three, four and five-combinations of the 36 cases were generated without repetition obtaining respectively 630, 7140, 58905 and 376992 characterization groups.

Fitting performance factors for flux and rejection were defined to evaluate the characterization groups created under the assumption that the more the variation of the characterization group correlates with that of the control group, the better the prediction of the behavior is expected.

The medium square difference of permeate flux (MSDJ) was defined (Eq. (3)) from the difference between the calculated fluxes obtained using every one of the $N P=93$ different parameters sets and the permeate flux value obtained for the reference set of parameters $\left(\mathrm{J}_{\mathrm{v}}^{\circ}\right)$.

$M S D J=\frac{\sqrt{\sum_{n=1}^{N P}\left(J_{v, n}-J_{v}^{\circ}\right)^{2}}}{N}$

Then, the fitting performance of permeate flux (FPJ) for a characterization group $k$ was defined (Eq. (4)) as the coefficient of determination between the MSDJ factor calculated for the characterization group and the $M S D J$ for the control group $\left(M_{S D J}\right)$. 
$F P J_{k}=\left(\frac{\operatorname{Cov}\left(M S D J_{k}, M S D J_{c g}\right)}{\sqrt{\operatorname{Cov}\left(M S D J_{k}, M S D J_{k}\right) \cdot \operatorname{Cov}\left(M S D J_{c g}, M S D J_{c g}\right)}}\right)^{2}$

Seemingly, MSDR (Eq. (5)) is defined as the medium square difference for rejection which is calculated taken into account the differences on rejection of all NC components using the random parameter sets and the reference parameter set $\left(R_{i}^{o}\right)$. The fitting performance (FPR) for rejection is also defined from the coefficient of determination between the MSDR factor calculated for each characterization group and that for the control group (MSDR $\left.{ }_{\mathrm{cg}}\right)(\mathrm{Eq}$. (6)).

$M S D R_{k}=\frac{\sqrt{\sum_{n=1}^{N P} \sum_{i=1}^{N C}\left(R_{i, n}-R_{i}^{\circ}\right)^{2}}}{N}$
$F P R_{k}=\left(\frac{\operatorname{Cov}\left(M S D R_{k}, M S D R_{c g}\right)}{\sqrt{\operatorname{Cov}\left(M S D R_{k}, M S D R_{k}\right) \cdot \operatorname{Cov}\left(M S D R_{c g}, M S D R_{c g}\right)}}\right)^{2}$

\subsection{Experimental methodology}

\subsubsection{Experimental set up for nanofiltration experiments}

The nanofiltration experiments were carried out in the pilot plant presented in Fig. 2 which was equipped with the polymeric membrane ESNA1-LF2 commercialized by Hydranautics displayed in a flat module with an effective area of $37.84 \mathrm{~cm}^{2}$. Both permeate and retentate streams were recirculated into the feed tank in order to keep a constant feed concentration. Applied pressure was fixed with two valves and controlled with three manometers upstream, downstream and on the membrane module.

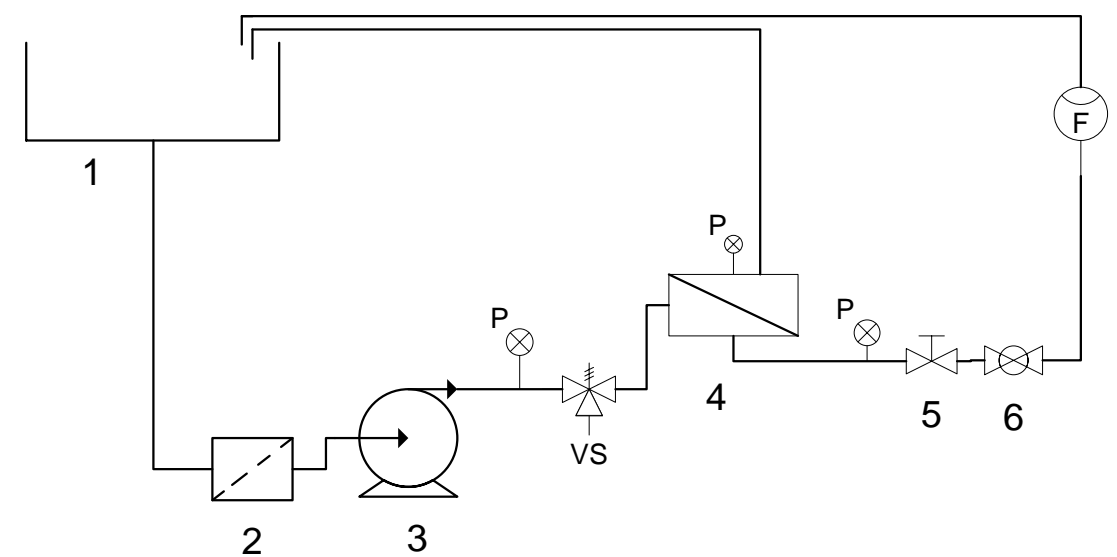

Fig. 2. Experimental nanofiltration set-up.

1. Feed tank. 2. Filter. 3. Pump. 4. Membrane module. 5 and 6. Valves.

Feed solutions were prepared with deionized water and pure grade salts, fixing its temperature on $25{ }^{\circ} \mathrm{C}$ with a heat exchanger / refrigerator. The $\mathrm{pH}$ of the solution was set on a value of 6 , adding $\mathrm{NaOH}$ or $\mathrm{HCl}$ if it were necessary. The effect of concentration polarization was reduced using a turbulence promoter in the membrane feed side and by setting the cross-flow velocity at $2 \mathrm{~m} / \mathrm{s}$.

\subsubsection{Rejection experiments}

Different feed compositions of different complexity were tested: solutions of single salts and mixtures of two and three salts. As single salt experiments, the rejection of $\mathrm{NaCl}, \mathrm{NaNO}_{3}, \mathrm{Na}_{2} \mathrm{SO}_{4}, \mathrm{CaCl}_{2}$, $\mathrm{Ca}\left(\mathrm{NO}_{3}\right)_{2}, \mathrm{MgCl}_{2}, \mathrm{Mg}\left(\mathrm{NO}_{3}\right)_{2}$ and $\mathrm{MgSO}_{4}$ was studied. Total concentration of 10, 25, 40, 55 and $70 \mathrm{mN}$ were tested at pressures of 5 and 10 bar. 
Binary salts mixtures with three different ions were also fed onto the membrane with combinations of the salts using in single salts experiences except the calcium ones. Equinormal compositions were set for the cations with the experiments with a common anion and vice versa. Two levels of total concentration of 40 and $70 \mathrm{mN}$ were tested at 5 and 10 bar.

For ternary salts mixtures only the cases with one cation and the three anions were experimentally tested with a total concentration of 40 and $70 \mathrm{mN}$ for the cation and equinormal composition for the anions at the effective pressures of 5 and 10 bar.

A validation case with a mixture of all the ions was used to evaluate the validity of the model. A feed solution with a total concentration of $50 \mathrm{mN}$ was prepared with the following composition expressed as percentage of equivalents: $20 \% \mathrm{NaCl}, 30 \% \mathrm{NaNO} 3$ and 50\% MgSO4. Five levels of applied pressure were studied: 5, 7, 9.5, 12 and 13 bar. All the experiences are summarized in Table 5.

The experiment was performed until stabilization of permeate flux and conductometric measures was observed. Complete ions analyses were performed for feed and permeate by ion chromatography.

Table 5. Summary of the present salts, equivalent fractions of each salt $\left(x_{i}\right)$, total concentration $\left(C_{T}\right)$ and effective pressure for the experimental experiences (* applied pressure).

\begin{tabular}{|c|c|c|c|c|}
\hline & Salts & $\mathbf{X}_{1}-\mathbf{X}_{2}-\mathbf{X}_{3}$ & $\begin{array}{c}\mathrm{C}_{\mathrm{T}} \\
(\mathrm{mN})\end{array}$ & $\begin{array}{c}\mathbf{P} \\
\text { (bar) }\end{array}$ \\
\hline Single salts & $\begin{array}{c}\mathrm{NaCl} \\
\mathrm{NaNO}_{3} \\
\mathrm{Na}_{2} \mathrm{SO}_{4} \\
\mathrm{MgCl}_{2} \\
\mathrm{Mg}\left(\mathrm{NO}_{3}\right)_{2} \\
\mathrm{MgSO}_{4} \\
\mathrm{CaCl}_{2} \\
\mathrm{Ca}\left(\mathrm{NO}_{3}\right)_{2} \\
\end{array}$ & & $\begin{array}{l}10 \\
25 \\
40 \\
55 \\
70\end{array}$ & $\begin{array}{c}5 \\
10\end{array}$ \\
\hline $\begin{array}{l}\text { Binary mixtures } \\
\text { (common cation) }\end{array}$ & 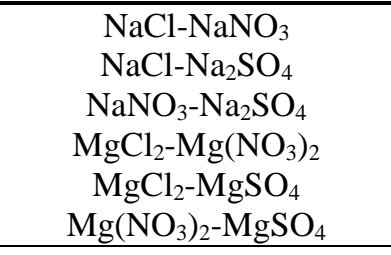 & $0.5-0.5$ & $\begin{array}{l}40 \\
70\end{array}$ & $\begin{array}{c}5 \\
10\end{array}$ \\
\hline $\begin{array}{l}\text { Binary mixtures } \\
\text { (common anion) }\end{array}$ & $\begin{array}{c}\mathrm{NaCl}-\mathrm{MgCl}_{2} \\
\mathrm{NaNO}_{3}-\mathrm{Mg}\left(\mathrm{NO}_{3}\right)_{2} \\
\mathrm{Na}_{2} \mathrm{SO}_{4}-\mathrm{MgSO}_{4}\end{array}$ & $0.5-0.5$ & $\begin{array}{l}40 \\
70\end{array}$ & $\begin{array}{c}5 \\
10\end{array}$ \\
\hline $\begin{array}{l}\text { Ternary mixtures } \\
\text { (common cation) }\end{array}$ & $\begin{array}{c}\mathrm{NaCl}-\mathrm{NaNO}_{3}-\mathrm{Na}_{2} \mathrm{SO}_{4} \\
\mathrm{MgCl}_{2}-\mathrm{Mg}\left(\mathrm{NO}_{3}\right)_{2}-\mathrm{MgSO}_{4}\end{array}$ & $0.33-0.33-0.33$ & $\begin{array}{l}40 \\
70\end{array}$ & $\begin{array}{c}5 \\
10\end{array}$ \\
\hline Validation case & $\mathrm{NaCl}-\mathrm{NaNO}_{3}-\mathrm{MgSO}_{4}$ & $0.2-0.3-0.5$ & 50 & $\begin{array}{c}5\left(^{*}\right) \\
7\left(^{*}\right) \\
9.5\left(^{*}\right) \\
12\left(^{*}\right) \\
13\left({ }^{*}\right)\end{array}$ \\
\hline
\end{tabular}

\section{Results and discussion}

\subsection{Evaluation of the characterization groups obtained with simulation}

Characterization groups formed by two, three, four and five cases were built from the single cases shown in Table 6 and calculated for all parameter sets.

The fitting performance factor for permeate flux (FPJ) showed good values near to 1 in all simulated cases. FPJ was over 0.99 in $69 \%$ of the characterization groups and over 0.80 in the $100 \%$ of the cases. Combination of cases with FPJ values smaller than 0.99 were discarded for the posterior study.

The fitting performance for rejection results showed that good grades of fitting performance for rejection (FPR near to 1) can be achieved even for combinations using a low number of cases. For all groups, the FPR values fell between 0.85 and 0.90. As an example, Fig. 3 shows the FPR values for the 
characterization groups formed by the 4937 selected three-cases combinations which have FPJ values greater than 0.99 . The observed distribution of the FPR was similar in all the cases, regardless the size of the group used for the calculation. This may suggest that there are some combination of cases offering better information in the prediction of the control group. According to the results, total rejection was chosen as the decision variable upon which study the grade of correlation between the control group and the characterization groups. The best characterization groups corresponding to high FPR values are shown in Table 9. It is remarkable that the same single cases are repeated in almost all the groups, so analyzing their feed composition and operating conditions will determine the best experimental conditions to have a good knowledge of the nanofiltration behavior of the control group. To make possible a comparison, the groups with lower FPR values for the two, three, four and five combinations were selected too and shown in Table 9.

The characteristics of the cases forming part of these groups that lead to the best and worst correlation can be seen in Table 6. The principal difference between them are the chosen salts for each group. It is noted that the groups where $\mathrm{MgSO}_{4}$ forms part of the feed has lower FPR and $\mathrm{Na}_{2} \mathrm{SO}_{4}$ seems to be the better choice among the sulfate salts. It is also remarkable that some characterization sets with only two single salt also showed good correlation.

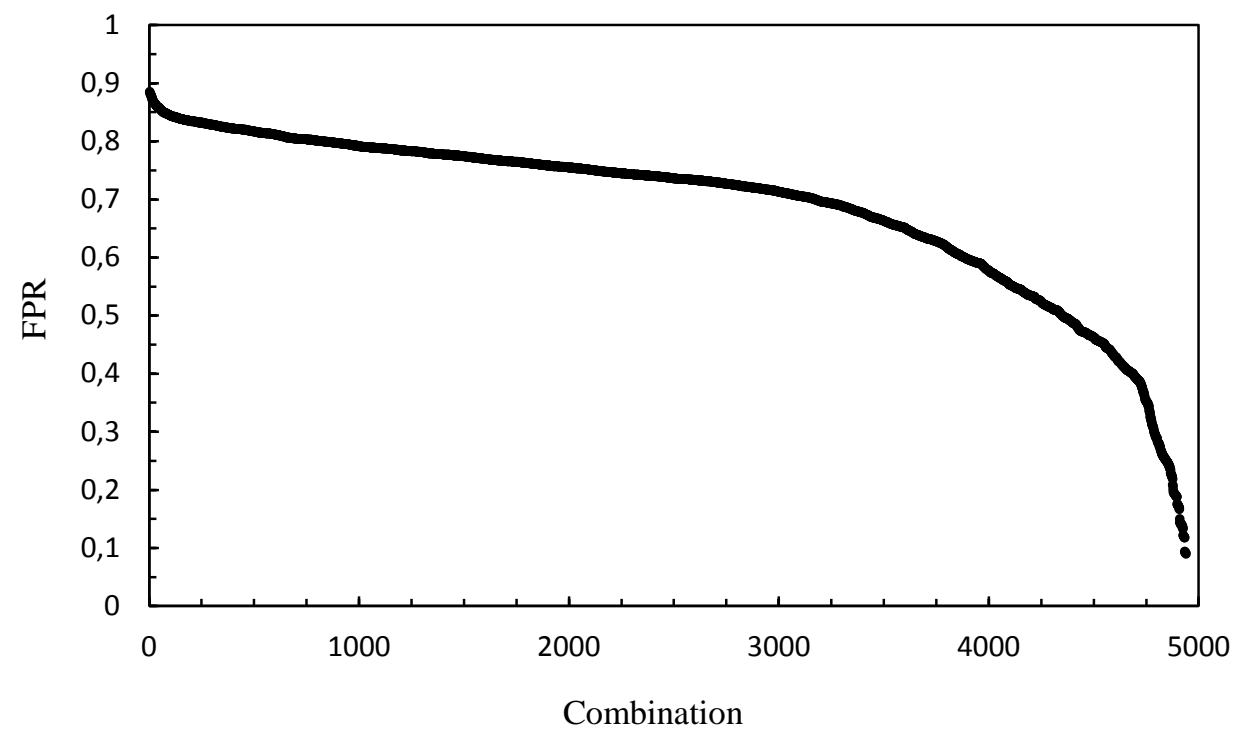

Fig. 3. Fitting performance values for rejection (FPR) for the three-cases combinations with fitting performance for permeate flux (FPJ) $>0.99$.

Table 6. Characterization groups with the higher and lower fitting performance factors for rejection (FPR) and their performance factors for permeate flux (FPJ).

\begin{tabular}{cccc|cccc}
\hline $\begin{array}{c}\text { Number } \\
\text { of cases }\end{array}$ & Cases & FPR & FPJ & $\begin{array}{c}\text { Number } \\
\text { of cases }\end{array}$ & Cases & FPR & FPJ \\
\hline 2 & 1136 & 0.846 & 0.9998 & 2 & 1623 & 0.089 & 0.9829 \\
3 & 111936 & 0.886 & 0.9986 & 3 & 162334 & 0.089 & 0.9926 \\
4 & 10111936 & 0.892 & 0.9994 & 4 & 7162334 & 0.092 & 0.9959 \\
5 & 411193536 & 0.898 & 0.9995 & 5 & 716232534 & 0.095 & 0.9975 \\
\hline
\end{tabular}

The lognormal distribution fitted well the values of the difference (1 - FPR) (Table 7). Fig. 4 shows the results for each model group with combinations of two, three, four and five cases. According to this distribution $0.12 \%$ of the two and three-cases combination groups, $0.08 \%$ and $0.04 \%$ of the four and fivecases characterization groups, respectively showed values of FPR greater than 0.9. 
Table 7. Log-normal distribution of the difference (1- FPR).

\begin{tabular}{ccccccc}
\hline $\begin{array}{c}\text { Number } \\
\text { of cases }\end{array}$ & $\begin{array}{c}\text { Mean } \\
\text { value }\end{array}$ & Variance & $\boldsymbol{\mu}$ & $\boldsymbol{\sigma}$ & $\begin{array}{c}\text { Standard } \\
\text { error }(\boldsymbol{\mu})\end{array}$ & $\begin{array}{c}\text { Standard } \\
\text { error }(\boldsymbol{\sigma})\end{array}$ \\
\hline 2 & 0.36 & 0.022 & -1.09 & 0.40 & $1.6 \cdot 10^{-2}$ & $1.1 \cdot 10^{-2}$ \\
3 & 0.31 & 0.013 & -1.22 & 0.36 & $4.2 \cdot 10^{-3}$ & $3.0 \cdot 10^{-3}$ \\
4 & 0.29 & 0.008 & -1.30 & 0.32 & $1.3 \cdot 10^{-3}$ & $9.3 \cdot 10^{-4}$ \\
5 & 0.28 & 0.006 & -1.36 & 0.28 & $4.6 \cdot 10^{-4}$ & $3.3 \cdot 10^{-4}$ \\
\hline
\end{tabular}

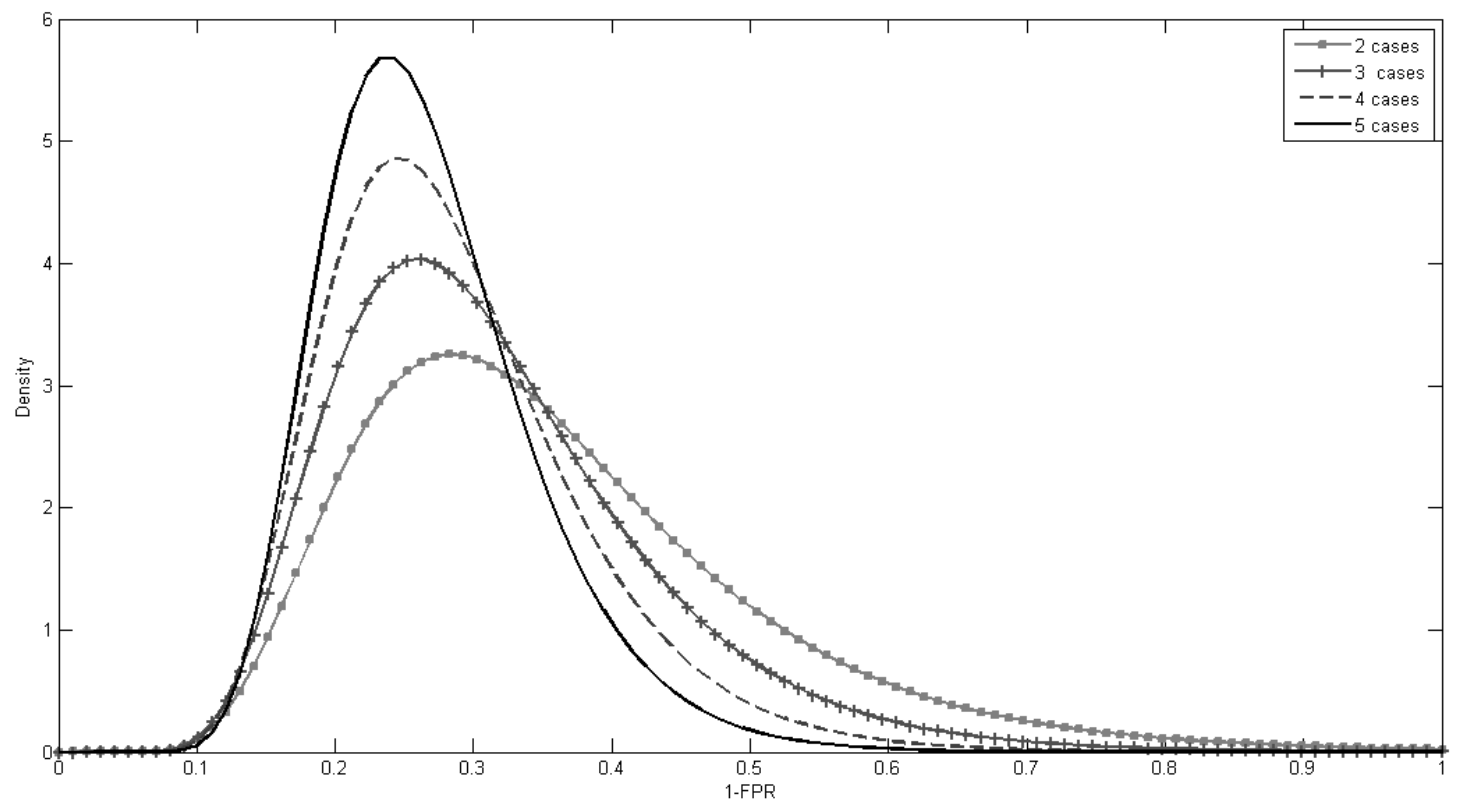

Fig. 4. Probability density function for (1 - FPR) corresponding to characterization groups formed with combinations of two, three, four and five cases.

\subsection{Experimental validation of the characterization groups}

To check the previous results obtained with simulation, the DSPM-DE model was fitted with the optimal characterization groups obtained from the simulation study and, then, the flux and total rejection for the validation case were calculated with these parameters. A case with a low correlation index was performed in order to know if there are differences between the results obtained fitting with an optimum characterization group and with a bad combination of cases. As there was more experimental data available (Table 7), the model was also fitted with all the experimental cases (127) in order to know if the hypothesis that a good prediction of the nanofiltration behavior can be achieved with a reduced number of experiments is true. In Fig. 5 and Fig. 6 prediction of both the permeate flux and the total rejection with different groups of experiments is shown. 


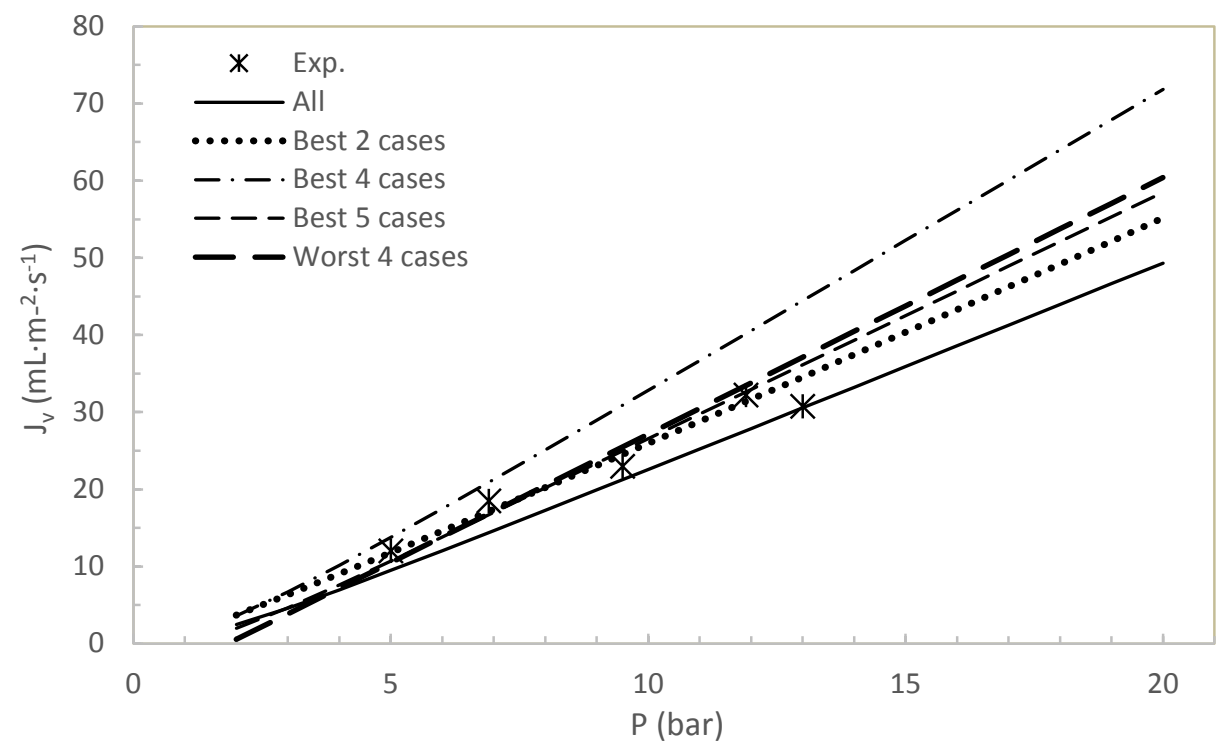

Fig. 5. Permeate flux for the validation case obtained experimentally and calculated with different model groups.

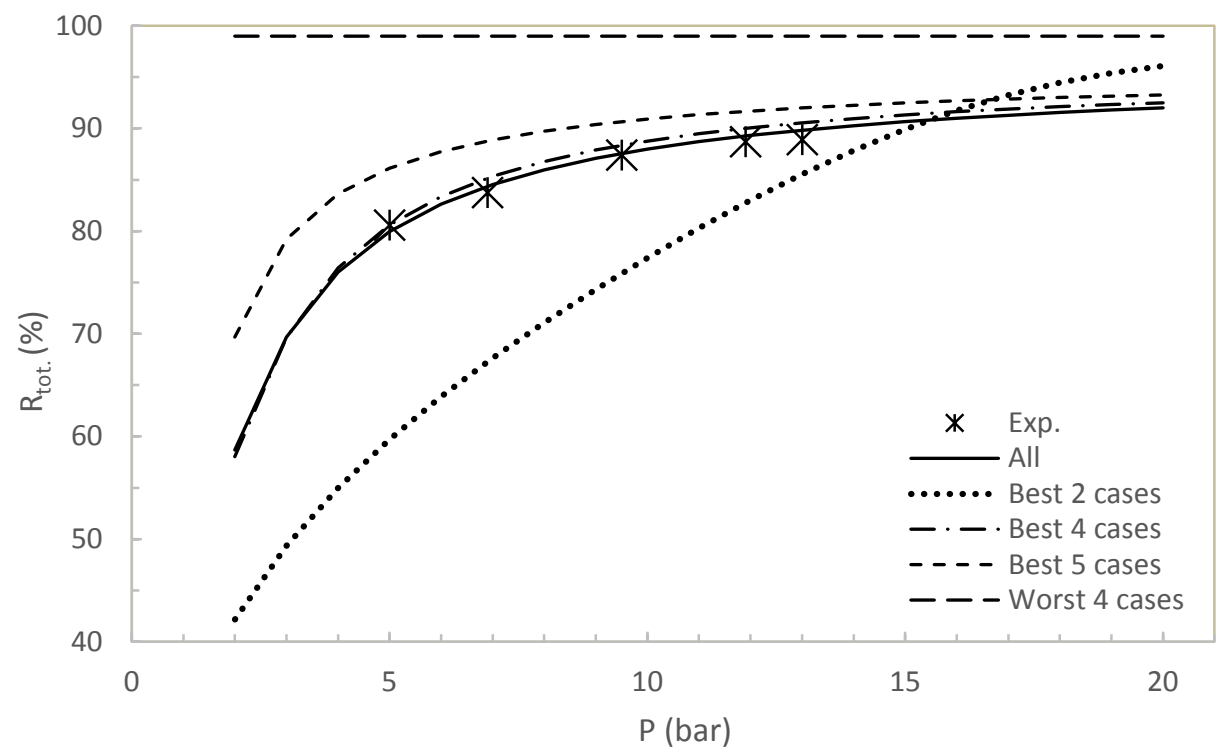

Fig. 6. Total rejection for the validation case obtained experimentally and calculated with different model groups.

It can be observed that a high number of experiments is not necessary to achieve an effective prediction, as with the four and five cases characterization group the prediction for the total rejection is as good as the obtained with the total of the experimental cases.

Moreover, it could be noticed that the presented procedure to select the best characterization groups it is a good way to discriminate between the operating conditions as there are remarkable differences between the obtained results with the optimal conditions and the worst groups.

The best results for the total rejection prediction does not agree with that for the permeate flux as the group selection was optimized for the total rejection variable. A future improvement for the selection procedure could be the simultaneous optimization of both variables. 


\section{Conclusions}

A huge number of simulations based on the comparison of different characterization groups with a control group of groundwaters could be generated using a program based on the DSPM-DE. These results allowed to define performance factors for flux and rejection. Fitting performance of flux was good for almost all characterization groups, but a small fraction of the characterization groups yielded higher fitting performance of rejection respect to the remaining groups. These characterization sets should be the preferred ones to be carried out when direct fitting from experiments is going to be performed.

The selected characterization groups were experimentally tested. The model fitted from these experiments resulted in a good prediction of a validation case. For the validation case used, a characterization group of four selected experiments was able to achieve a good fitting, not being observed substantially improvement by augmenting the number of experiments.

Therefore, it can be concluded that the present procedure is appropriate to determine the best experiments for a direct fitting of the DSPM-DE model. 


\section{Symbols}

\section{Latin}

$C_{T} \quad$ total feed composition, $\mathrm{mN}$

FPJ Fitting performance factor for permeate flux of a characterization group.

FPR Fitting performance factor for rejection of a characterization group.

$L_{p, w} \quad$ membrane permeability to pure water, $\mathrm{L} \cdot \mathrm{m}^{-2} \cdot \mathrm{h}^{-1} \cdot \mathrm{bar}^{-1}$

$N C \quad$ number of ions type in solution

NR number of random parameter sets generated using the Monte Carlo method

$R \quad$ Rejection, \%

$r_{p} \quad$ pore size of the membrane, $\mathrm{nm}$

$v \quad$ cross flow velocity, $\mathrm{m} / \mathrm{s}$

$x \quad$ fraction of equivalents

$T \quad$ feed temperature, ${ }^{\circ} \mathrm{C}$

\section{Greek symbols}

$\Delta x / A_{k} \quad$ ratio of membrane thickness to effective porosity, $\mu \mathrm{m}$

$\varepsilon \quad$ dielectric constant of the membrane

$\mu \quad$ dynamic viscosity, Pa·s

$\chi_{d} \quad$ membrane charge density, $\mathrm{Eq} \cdot \mathrm{mol}^{-1}$

$\chi_{\mathrm{dq}} \quad$ coefficient of potential correlation for membrane charge density, $\mathrm{Eq} \cdot \mathrm{mol}^{-1}$

$\chi$ ds exponent of potential correlation for membrane charge density

\section{Subscripts}

exp experimental

i ion component

cg control group

$k \quad$ characterization group

n $\quad$ Monte Carlo set

tot total

val validation

\section{Superscripts}

* reference value

\section{Acknowledgments}

The Spanish Ministry for Economy and Competitiveness (Project OPTIMEM CTM2010-20248) is kindly acknowledged. 


\section{References}

[1] W. R. Bowen and H. Mukhtar, "Characterisation and prediction of separation performance of nanofiltration membranes,” J. Memb. Sci., 112 (1996) 263-274.

[2] W. R. Bowen and J. S. Welfoot, "Modelling the performance of membrane nanofiltration critical assessment and model development,” Chem. Eng. Sci., 57 (2002) 1121-1137.

[3] J. A. Otero, O. Mazarrasa, J. Villasante, V. Silva, P. Prádanos, J. I. Calvo, and A. Hernández, "Three independent ways to obtain information on pore size distributions of nanofiltration membranes," J. Memb. Sci., 309 (2008) 17-27.

[4] N. S. Kotrappanavar, A. A. Hussain, M. E. E. Abashar, I. S. Al-Mutaz, T. M. Aminabhavi, and M. N. Nadagouda, "Prediction of physical properties of nanofiltration membranes for neutral and charged solutes,” Desalination, 280 (2011) 174-182.

[5] J. Straatsma, G. Bargeman, H. C. van der Horst, and J. a. Wesselingh, "Can nanofiltration be fully predicted by a model?,” J. Memb. Sci., 198 (2002) 273-284.

[6] I. G. y M. de España, “Sistema de información del agua subterránea,” 2014. [Online]. Available: www.igme.es.

[7] S. Wilks, "Determination of sample sizes for setting tolerance limits,” Ann. Math. Stat., 96 (1941) 91-96.

[8] J. Luo and Y. Wan, "Effect of highly concentrated salt on retention of organic solutes by nanofiltration polymeric membranes,” J. Memb. Sci., 372 (2011) 145-153.

[9] G. Bargeman, J. M. Vollenbroek, J. Straatsma, C. G. P. H. Schroën, and R. M. Boom, "Nanofiltration of multi-component feeds. Interactions between neutral and charged components and their effect on retention,” J. Memb. Sci., 247 (2005) 11-20.

[10] A. I. Cavaco Morão, A. Szymczyk, P. Fievet, and A. M. Brites Alves, "Modelling the separation by nanofiltration of a multi-ionic solution relevant to an industrial process,” J. Memb. Sci., 322 (2008) 320-330.

[11] M. Diná Alfonso, G. Hagmeyer, and R. Gimbel, "Streaming potential measurements to assess the variation of nanofiltration membranes surface charge with the concentration of salt solutions," Sep. Pu 22-23 (2001) 529-541.

[12] A. A. Hussain, S. K. Nataraj, M. E. E. Abashar, I. S. Al-Mutaz, and T. M. Aminabhavi, "Prediction of physical properties of nanofiltration membranes using experiment and theoretical models," J. Memb. Sci., 310 (2008) 321-336.

[13] N. C. Nguyen, S.-S. Chen, H.-T. Hsu, and C.-W. Li, "Separation of three divalent cations $\left(\mathrm{Cu}^{2+}\right.$, $\mathrm{Co}^{2+}$ and $\mathrm{Ni}^{2+}$ ) by NF membranes from pHs 3 to 5,” Desalination, 328 (2013) 51-57.

[14] D.-X. Wang, X.-L. Wang, Y. Tomi, M. Ando, and T. Shintani, "Modeling the separation performance of nanofiltration membranes for the mixed salts solution,” J. Memb. Sci., 280 (2006) 734-743.

[15] D. Nanda, K.-L. Tung, C.-C. Hsiung, C.-J. Chuang, R.-C. Ruaan, Y.-C. Chiang, C.-S. Chen, and T.-H. Wu, "Effect of solution chemistry on water softening using charged nanofiltration membranes," Desalination, 234 (2008) 344-353.

[16] Y.-L. Lin, P.-C. Chiang, and E.-E. Chang, "Removal of small trihalomethane precursors from aqueous solution by nanofiltration,” J. Hazard. Mater., 146 (2007) 20-29. 